\title{
T-SUPERIORITY AND t-NORM-BASED IMAGES OF FUZZY SETS
}

\author{
Slavka Bodjanova - Martin Kalina
}

\begin{abstract}
Comparisons of finite fuzzy sets based on the results of conjunctive and disjunctive aggregations of their membership grades are studied. For a given t-norm $T$, the notion of $T$-superiority of fuzzy sets is introduced. When a fuzzy set $g$ is $T$-superior to a fuzzy set $f$, the description of a vague concept by $g$ is much more desirable than the description by $f$. For a comparison of a fuzzy set $f$ with the "standard" fuzzy set $g$ (describing the desirable or historically the most common characterization of the vague concept in question) t-norm-based transformations of $f$ with respect to $g$, called the $T$-images of $f$, are suggested. A special type of the $T$-image of $f$ with respect to $g$ may be considered as a soft evaluation of $T$-superiority of $g$ to $f$.
\end{abstract}

\section{Introduction}

The task of analyzing vague data described by fuzzy sets is very broad and heterogeneous [4], 7], [10]. We focus on comparison of finite fuzzy sets. Since a fuzzy set describes vagueness with membership grades in the range $[0,1]$, comparison of fuzzy sets may be based on aggregation of their membership grades. We refer to aggregation by special type of aggregation functions called triangular norms (t-norms) and their duals, triangular conorms (t-conorms) [1], [3], [6]. Each t-norm is a generalization of the logical conjunction from the two-valued set $\{0,1\}$ to the whole unit interval. On the other hand, a t-conorm generalizes the logical disjunction. The result of a conjunctive or a disjunctive aggregation may represent "the worst" or "the best" case scenario, depending on whether the highest or the lowest value of a particular aggregation is preferred. Under certain circumstances, if the worst case scenario obtained from a fuzzy set $g$ is better than the best case scenario derived from a fuzzy set $f$, we may claim that $g$ is superior to $f$. Let us consider the following illustrative example.

(c) 2016 Mathematical Institute, Slovak Academy of Sciences. 2010 Mathematics Subject Classification: 03E72, 90 B50.

Keywords: coarser images of fuzzy sets, comparison of fuzzy sets, t-norm.

The work of Martin Kalina has been supported from the Science and Technology Assistance Agency under the contract No. APVV-14-0013, and from the VEGA grant agency, grant number 2/0069/16. 
ExAmple 1 . Let $X=\left\{x_{1}, x_{2}, x_{3}, x_{4}\right\}$ be a set of four online homework assignments assigned to students in a college class. Denote by $f$ the concept "progress on $X$ ". Let the progress of each student be evaluated by the proportion of problems in a homework $x_{i} \in X, i=1, \ldots, 4$, that the student solved correctly. Data for two students, say Anton $\left(f_{A}\right)$ and Benjamin $\left(f_{B}\right)$, are given in Table 1 . Clearly, Anton's progress is superior to Benjamin's. He missed only one homework, while Benjamin missed two. Moreover, Anton's worst (minimal) result 0.5 is better (higher) than Benjamin's best (maximal) result 0.2.

TABle 1. Progress on $X$ characterized by $f$.

\begin{tabular}{|c||c|c|c|c|}
\hline$f$ & $x_{1}$ & $x_{2}$ & $x_{3}$ & $x_{4}$ \\
\hline \hline$f_{A}$ & 0 & 0.8 & 0.5 & 0.85 \\
\hline$f_{B}$ & 0.2 & 0.1 & 0 & 0 \\
\hline
\end{tabular}

The conclusions in Example 1 were based on the results of aggregation of available data by the t-norm $T_{M}$ (minimum) and its dual t-conorm $S_{M}$ (maximum). Depending on the data and the purpose of study, other aggregation functions can be considered [1], [3], [5]. The first goal of our paper is to provide a mathematical description of the "superiority" relationship between two fuzzy sets. For a given t-norm $T$ and a pair of fuzzy sets $f$ and $g$ we state when $g$ is "T-superior" to $f$. Because $T$-superiority is a rare event, we also introduce partial $T$-superiority and a measure of $T$-superiority.

In some applications a comparison of several evaluations of the same concept with so-called "standard" description of the concept may be required. For example, in our illustrative Example 1, we may have information about the "standard" progress on $X$ estimated from the data on all students taking the same set of homework in the past. The task is to decide whether two students evaluated, e.g., by fuzzy sets $f_{C}$ and $f_{D}$, show more or less the same progress when compared with the standard progress represented by a fuzzy set $g$. Instead of the detailed pointwise comparison of the membership grades of $f_{C}$ and $f_{D}$ (which may lead to a high computational cost) we suggest a comparison of coarser transformations (images) of $f_{C}$ and $f_{D}$ based on $g$. Coarsening of fuzzy sets is often used in applications where a complex vague structure needs to be simplified in order to provide easier manipulation and interpretation of data. Well-known examples of coarser transformations of fuzzy sets are $\alpha$-level sets [4], 7] or fuzzy $\alpha$-level sets [9], where coarsening depends on a single parameter $\alpha$. Coarsening of fuzzy sets with respect to more parameters was studied in [2].

The second goal of our paper is to propose some transformations (preferably coarser images) of fuzzy sets with respect to a given "standard" fuzzy set $g$ whose membership grades reflect a t-norm-based relationship between evaluated fuzzy 


\section{T-SUPERIORITY AND t-NORM-BASED IMAGES OF FUZZY SETS}

sets and the given set $g$. We introduce the $T$-image of a fuzzy set $f$ with respect to $g$ based on the results of aggregation of subsets of membership grades of $g$ by a t-norm $T$. A special type of the $T$-image of $f$ with respect to $g$ may be considered as a soft evaluation of $T$-superiority of $g$ to $f$. The choice of $T$ depends on the area of application and on the purpose of study.

Our paper is organized as follows. After some preliminary notions and notations in Section 2, the concept of $T$-superiority and partial $T$-superiority of fuzzy sets is introduced in Section 3. T-images of fuzzy sets are presented in Section 4. Concluding remarks are in Section 5.

\section{Preliminary notions}

A vague concept on a set $X$ is often described by a mapping $f: X \rightarrow[0,1]$ known as a fuzzy set. In our study we assume that $X$ is a finite universe of discourse. We denote by $\mathscr{F}(X)$ the family of all fuzzy sets on $X$ and by $|X|$ the cardinality of $X$. The meet and join operators on $\mathscr{F}(X)$ are given, respectively, by $(f \wedge g)(x)=\min \{f(x), g(x)\}$ and $(f \vee g)(x)=\max \{f(x), g(x)\}$, for all $x \in X$. Then $(\mathscr{F}(X), \wedge, \vee, \mathbf{0}, \mathbf{1})$ is a complete and distributive lattice, where $\mathbf{0}$ and $\mathbf{1}$ are constant fuzzy sets, representing, respectively, the empty set and the set $X$. Obviously, $f \leq g$ if and only if $f(x) \leq g(x)$ for all $x \in X$. The complement of $f$ is the fuzzy set $f^{c}$ defined pointwise by $f^{c}(x)=1-f(x)$.

The support of $f$ is the set supp $f=\{x \in X ; f(x)>0\}$. The set

$$
\Lambda_{f}=\{a \in(0,1] ; f(x)=a \text { for some } x \in \operatorname{supp} f\}
$$

will be called the level set of $f$. When $\left|\Lambda_{f}\right|<\left|\Lambda_{g}\right|$ we say that $f$ is coarser than $g$.

Comparison of vague concepts described by fuzzy sets may be realized by comparison of the results of aggregation of their membership grades. Recall that an aggregation function with arity $n$ is an increasing mapping $A g:[0,1]^{n} \rightarrow[0,1]$ such that $A g(0, \ldots, 0)=0$ and $A g(1, \ldots, 1)=1$. For $n=2$ we obtain a binary aggregation function. An associative and commutative binary aggregation function $T$ is called a triangular norm (t-norm) if it satisfies $T(a, 1)=a$ for all $a \in[0,1]$. An element $a \neq 0$ is called a zero divisor of $T$ if there exists $b \neq 0$ such that $T(a, b)=0$. We say that a t-norm $T_{1}$ is stronger than a t-norm $T_{2}$, with the notation $T_{2} \leq T_{1}$, if $T_{2}(a, b) \leq T_{1}(a, b)$ for all $(a, b) \in[0,1]^{2}$. Because of associativity, the arity of t-norms can be generalized to any $n>2$. For aggregation of elements $\left(a_{1}, \ldots, a_{n}\right) \in[0,1]^{n}$ we will consider the following t-norms:

the minimum

$$
T_{M}\left(a_{1}, \ldots, a_{n}\right)=\min \left(a_{1}, \ldots, a_{n}\right),
$$

the Lukasiewicz t-norm

$$
T_{L}\left(a_{1}, \ldots, a_{n}\right)=\max \left(0, \sum_{i=1}^{n} a_{i}-(n-1)\right),
$$


and the product t-norm

$$
T_{P}\left(a_{1}, \ldots, a_{n}\right)=\prod_{i=1}^{n} a_{i} .
$$

Then $T_{L} \leq T_{p} \leq T_{M}$ and $T \leq T_{M}$ for any t-norm $T$. Note that for arbitrary $a_{n+1} \in[0,1]$

$$
T\left(a_{1}, \ldots a_{n}, a_{n+1}\right) \leq T\left(a_{1}, \ldots, a_{n}\right) .
$$

The dual t-conorm of a t-norm $T:[0,1]^{2} \rightarrow[0,1]$ is the mapping $S:[0,1]^{2} \rightarrow[0,1]$ defined by

$$
S(a, b)=1-T(1-a, 1-b) .
$$

Then $S$ is an associative and commutative binary aggregation function that satisfies $S(a, 0)=a$ for all $a \in[0,1]$. The t-conorms of arity $n$ dual of $T_{M}, T_{L}$ and $T_{P}$, are, respectively, the maximum

$$
S_{M}\left(a_{1}, \ldots, a_{n}\right)=\max \left(a_{1}, \ldots, a_{n}\right),
$$

the Eukasiewicz t-conorm

$$
S_{L}\left(a_{1}, \ldots, a_{n}\right)=\min \left(1, \sum_{i=1}^{n} a_{i}\right),
$$

and the probabilistic sum

$$
S_{P}\left(a_{1}, \ldots, a_{n}\right)=1-\prod_{i=1}^{n}\left(1-a_{i}\right) .
$$

Then $S_{M} \leq S_{P} \leq S_{L}$ and $S_{M} \leq S$ for any t-conorm $S$. For arbitrary $a_{n+1} \in[0,1]$

$$
S\left(a_{1}, \ldots a_{n}, a_{n+1}\right) \geq S\left(a_{1}, \ldots, a_{n}\right) .
$$

For $a \in[0,1]$ we use the convention $T(a)=S(a)=a$.

\section{T-superiority of fuzzy sets}

In this section, we introduce a comparison of fuzzy sets based on the results of conjunctive and disjunctive aggregations of their membership grades.

Definition 1. Consider a t-norm $T$, its dual t-conorm $\mathrm{S}$ and a relation $R_{T}: \mathscr{F}(X) \times \mathscr{F}(X) \rightarrow\{0,1\}$ defined for all $(f, g) \in \mathscr{F}(X) \times \mathscr{F}(X)$ by $R_{T}(f, g)=1$ if $S(\{f(x) ; x \in \operatorname{supp} f\})<T(\{g(x) ; x \in \operatorname{supp} g\})$ and $|\operatorname{supp} f| \leq|\operatorname{supp} g|$, otherwise $R_{T}(f, g)=0$.

If $R_{T}(f, g)=1$, we say that $g$ is $T$-superior to $f$.

Note that the relation $R_{T}$ is an irreflexive, asymmetric and transitive relation on $\mathscr{F}(X) \backslash\{\boldsymbol{0}\}$. If for a fuzzy set $f \in \mathscr{F}(X)$ we have that $f(x)=1$ for some $x \in X$, then there is no fuzzy set $g \in \mathscr{F}(X)$ such that $R_{T}(f, g)=1$. 


\section{T-SUPERIORITY AND t-NORM-BASED IMAGES OF FUZZY SETS}

Remark 1. The support of the constant fuzzy set $\mathbf{0}$ is empty, i.e., supp $\mathbf{0}=\emptyset$. According to the convention, $S(\{f(x) ; x \in \emptyset\})=0$ and $T(\{f(x) ; x \in \emptyset\})=1$. Therefore, $\mathbf{0}$ is the only fuzzy set that is $T$-superior to itself, i.e., $R_{T}(\mathbf{0}, \mathbf{0})=1$.

$T$-superiority represents a rather rare relationship. When an additional element is included in aggregation, the numerical value of $T$-aggregation may decrease while the numerical value of $S$-aggregation may increase. Therefore, $R_{T}(f, g)=1$ is more likely when both $|\operatorname{supp} f|$, and $|\operatorname{supp} g|$ are small.

Now we address the relationship between $T$-superiority of $f$ and $g$ and $T$-superiority of their complements.

Proposition 1. Let $f, g \in \mathscr{F}(X)$ and $0<g(x)<1$ for all $x \in X$. Then $R_{T}(f, g)=1$ if and only if $R_{T}\left(g^{c}, f^{c}\right)=1$.

Proof. When $0<g(x)<1$ for all $x \in X$, then also $0<g^{c}(x)<1$ for all $x \in X$ and $|\operatorname{supp} g|=\left|\operatorname{supp} g^{c}\right|=|X|$. The relationship $R_{T}(f, g)=1$ implies that $f(x)<1$ for all $x \in X$, which means that $0<f^{c}(x)$ for all $x \in X$ and $\left|\operatorname{supp} f^{c}\right|=|X|$. Therefore $\left|\operatorname{supp} g^{c}\right| \leq\left|\operatorname{supp} f^{c}\right|$. Then $R_{T}(f, g)=1$ if and only if

$$
S(\{f(x) ; x \in \operatorname{supp} f\})<T(\{g(x) ; x \in \operatorname{supp} g\}) .
$$

However,

$$
S(\{f(x) ; x \in \operatorname{supp} f\})=1-T(\{1-f(x) ; x \in \operatorname{supp} f\})
$$

and

$$
T(\{g(x), x \in \operatorname{supp} g\})=1-S(\{1-g(x), x \in \operatorname{supp} g\}) .
$$

Inequality (1) can be rewritten as

$$
S(\{1-g(x), x \in \operatorname{supp} g\})<T(\{1-f(x) ; x \in \operatorname{supp} f\}),
$$

which is equivalent to

$$
S\left(\left\{g^{c}(x) ; x \in \operatorname{supp} g^{c}\right\}\right)<T\left(\left\{f^{c}(x) ; x \in \operatorname{supp} f^{c}\right\}\right) .
$$

Therefore $R_{T}\left(g^{c}, f^{c}\right)=1$.

One can easily observe that $T$-superiority of $g$ to $f$ is possible only when $f$ and $g$ do not have any common nonzero membership grade. Under certain circumstances, $T$-superiority of $g$ to $f$ may imply that $f$ is a subset of $g$.

Proposition 2. Assume $f, g \in \mathscr{F}(X)$ and $R_{T}(f, g)=1$. Then

i) $\Lambda_{f} \cap \Lambda_{g}=\emptyset$,

ii) if $\operatorname{supp} f \subset \operatorname{supp} g$, then $f \leq g$,

iii) if $T$ has no zero-divisors, then $R_{T}(\mathbf{0}, g)=1$ for all $g$,

iv) if $T$ has no zero-divisors and $f(x)<1$ for all $x \in X$, then $R_{T}(f, \mathbf{1})=1$.

P r o of. i) Because $T \leq T_{M}$ and $S_{M} \leq S$, if $R_{T}(f, g)=1$, then $R_{T_{M}}(f, g)=1$. At the same time, $R_{T_{M}}(f, g)=1$ implies that $\max \left\{\alpha ; \alpha \in \Lambda_{f}\right\}<\min \left\{\beta ; \beta \in \Lambda_{g}\right\}$. Hence $\Lambda_{f} \cap \Lambda_{g}=\emptyset$. 
ii) Because $\max \{f(x), x \in \operatorname{supp} f\}<\min \{g(x), x \in \operatorname{supp} g\}$, for each $x \in$ supp $g$ we obtain that $f(x)<g(x)$. Since supp $f \subset \operatorname{supp} g$, if $g(x)=0$, then $f(x)=0$. Hence $f(x) \leq g(x)$ for all $x \in X$ which means that $f \leq g$.

Properties iii) and iv) are obvious.

Note that neither $\Lambda_{f} \cap \Lambda_{g}=\emptyset$ nor $f \leq g$ guarantee that $S(\{f(x) ; x \in \operatorname{supp} f\})<$ $T(\{g(x) ; x \in \operatorname{supp} g\})$.

Now we assume that $f, g \in \mathscr{F}(X),|\operatorname{supp} f| \leq|\operatorname{supp} g|$, and

$$
S(\{f(x) ; x \in \operatorname{supp} f\}) \geq T(\{g(x) ; x \in \operatorname{supp} g\}) .
$$

Therefore, $g$ is not $T$-superior to $f$. However, it may happen that $g$ is $T$-superior to a part of $f$ restricted only to elements with low membership grades $f(x)$. Further in the text, $f_{p}$ will denote the following restriction of $f$ to a $p$-element subset of supp $f$ with the lowest membership grades

$$
f_{p}=\left\{\left(x_{(1)}, f\left(x_{(1)}\right)\right),\left(x_{(2)}, f\left(x_{(2)}\right)\right), \ldots,\left(x_{(p)}, f\left(x_{(p)}\right)\right)\right\},
$$

where $x_{(1)}, x_{(2)} \ldots, x_{(p)}$ are reordered elements from supp $f$ such that $f\left(x_{(1)}\right) \leq$ $f\left(x_{(2)}\right) \leq \cdots \leq f\left(x_{(p)}\right)$.

Definition 2. Consider a t-norm $\mathrm{T}$ and $f, g \in \mathscr{F}(X)$. Let $|\operatorname{supp} f|=m$. We say that $g$ is partially $T$-superior to $f$ if $|\operatorname{supp} f| \leq|\operatorname{supp} g|$ and there exists $p \in\{1, \ldots, m\}$ such that $R_{T}\left(f_{p}, g\right)=1$.

Remark 2. When $g$ is $T$-superior to $f$ and $m=|\operatorname{supp} f|$, then for all $p \in\{1, \ldots, m\}$ we obtain that $R_{T}\left(f_{p}, g\right)=1$.

Now we will introduce a measure of $T$-superiority.

Definition 3. Assume a t-norm $T$. Let a mapping $\gamma_{T}: \mathscr{F}(X) \times \mathscr{F}(X) \rightarrow[0,1]$ satisfy the following conditions for all $(f, g) \in \mathscr{F}(X) \times \mathscr{F}(X)$ :

$\mathrm{C} 1: \gamma_{T}(f, g)=1$ if and only if $R_{T}(f, g)=1$,

C2: $\gamma_{T}(f, g)=0$ if and only if $\left(R_{T}\left(f_{p}, g\right)=0\right.$ for all $p \in\{1,2, \ldots,|\operatorname{supp} f|\}$ or $|\operatorname{supp} f|>|\operatorname{supp} g|)$,

C3: if $h \in \mathscr{F}(X),|\operatorname{supp} h|=|\operatorname{supp} f|$ and $h \leq f$, then $\gamma_{T}(h, g) \geq \gamma_{T}(f, g)$,

C4: if $h \in \mathscr{F}(X),|\operatorname{supp} h|=|\operatorname{supp} g|$ and $g \leq h$, then $\gamma_{T}(f, g) \leq \gamma_{T}(f, h)$.

Then $\gamma_{T}$ is a measure of $T$-superiority and $\gamma_{T}(f, g)$ evaluates the degree to which $g$ is $T$-superior to $f$.

Note that in Definition 3 conditions C3 and C4 characterize the desired monotonicity of a measure of $T$-superiority. The value of $\gamma_{T}(f, g)$ increases when the membership grades of $f$ decrease or when the membership grades of $g$ increase. 


\section{T-SUPERIORITY AND t-NORM-BASED IMAGES OF FUZZY SETS}

Proposition 3. Assume a t-norm T. Consider $f, g \in \mathscr{F}(X)$ such that $m=$ $|\operatorname{supp} f|$. Let $j^{*}=0$ if $R_{T}\left(f_{p}, g\right)=0$ for all $p \in\{1, \ldots, m\}$ or $|\operatorname{supp} f|>$ $|\operatorname{supp} g|$, otherwise $j^{*}=\max \left\{p ; R_{T}\left(f_{p}, g\right)=1\right\}$. Then the coefficient

$$
\delta_{T}(f, g)=\frac{j^{*}}{m}
$$

evaluates the degree of T-superiority of $g$ to $f$.

P r o of. Obviously, $\delta_{T}(f, g) \in[0,1]$ and $\delta_{T}$ satisfies the condition C2 from Definition 3. Further, $\delta_{T}(f, g)=1$ if and only if $j^{*}=m$. Clearly, this happens if and only if $R_{T}\left(f_{m}, g\right)=1$, which is equivalent to $R_{T}(f, g)=1$, and therefore the condition $\mathrm{C} 1$ holds. Now we verify conditions $\mathrm{C} 3$ and $\mathrm{C} 4$.

C3: Assume $h \leq f$ and $|\operatorname{supp} h|=|\operatorname{supp} f|=m$. Then for all $p \in\{1, \ldots, m\}$ : $S\left(h\left(x_{(1)}\right), \ldots, h\left(x_{(p)}\right)\right) \leq S\left(f\left(x_{(1)}\right), \ldots, f\left(x_{(p)}\right)\right)$. Hence

$$
s^{*}=\max \left\{p ; R_{T}\left(h_{p}, g\right)=1\right\} \geq \max \left\{p ; R_{T}\left(f_{p}, g\right)=1\right\}=j^{*} .
$$

Therefore

$$
\delta_{T}(f, g)=\frac{j^{*}}{m} \leq \frac{s^{*}}{m}=\delta_{T}(h, g) .
$$

C4: Assume $g \leq h$ and $|\operatorname{supp} g|=|\operatorname{supp} h|$. Then we have that $T(\{g(x)$; $x \in \operatorname{supp} g\}) \leq T(\{h(x) ; x \in \operatorname{supp} h\})$. Therefore

$$
s^{*}=\max \left\{p ; R_{T}\left(f_{p}, h\right)=1\right\} \geq \max \left\{p ; R_{T}\left(f_{p}, g\right)=1\right\}=j^{*} .
$$

Hence

$$
\delta_{T}(f, g)=\frac{j^{*}}{m} \leq \frac{s^{*}}{m}=\delta_{T}(f, h)
$$

Lemma 1. Consider the lattice $(\mathscr{F}(X), \wedge, \vee, \mathbf{0}, \mathbf{1})$, fuzzy sets $f, h, g \in \mathscr{F}(X)$ and the measure of $T$-superiority $\delta_{T}$. Then

i) $\delta_{T}(\mathbf{0}, g)=1$ for all $g \neq \mathbf{0}$, whenever $T$ has no zero-divisors,

ii) $\delta_{T}(\mathbf{1}, g)=0$ for all $g$.

ExAmple 2. Let $f_{A}$ and $f_{B}$ be the fuzzy sets from Example 11. Recall that the set of all nonzero membership grades of $f_{A}$ is $\{0.5,0.8,0.85\}$ and the set of all nonzero membership grades of $f_{B}$ is $\{0.1,0.2\}$. It is clear that $f_{A}$ is $T_{M^{-}}$ superior to $f_{B}$. When we aggregate the data by the t-norm $T_{L}$ and its dual t-conorm $S_{L}$, we obtain that $S_{L}(0.1,0.2)=0.3>T_{L}(0.5,0.8,0.85)=0.15$. Therefore, we conclude that $f_{A}$ is not $T_{L}$-superior to $f_{B}$. However, there is a part of the fuzzy set $f_{B}$ restricted to the membership grade 0.1 such that $S_{L}(0.1)=0.1<T_{L}(0.5,0.8,0.85)=0.15$. Therefore, $\delta_{T_{L}}\left(f_{B}, f_{A}\right)=1 / 2$, which means that $f_{A}$ is $T_{L}$-superior to $f_{B}$ to the degree $1 / 2$. 
LeMma 2. Assume a t-norm $T$ and an aggregation function $\mathrm{Ag}$ with no zero divisors. Consider $f, g \in \mathscr{F}(X) \times \mathscr{F}(X)$ such that $|\operatorname{supp} f|=m$. Let $j^{*}=0$ if $R_{T}\left(f_{p}, g\right)=0$ for all $p \in\{1, \ldots, m\}$, or $|\operatorname{supp} f|>|\operatorname{supp} g|$, otherwise $j^{*}=\max \left\{p: R_{T}\left(f_{p}, g\right)=1\right\}$. Then the coefficient

$$
\psi_{T}(f, g)= \begin{cases}0 & \text { if } j^{*}=0, \\ \frac{\operatorname{Ag}\left(f\left(x_{(1)}\right), \ldots, f\left(x_{\left(j^{*}\right)}\right)\right)}{\operatorname{Ag}\left(f\left(x_{(1)}\right), \ldots, f\left(x_{(m)}\right)\right)} & \text { otherwise, }\end{cases}
$$

evaluates the degree of $T$-superiority of $g$ to $f$.

In analysis of vague data, $T$-superiority can be used for identification of unusual characterizations (outliers) of a considered vague concept. When $g \in \mathscr{F}(X)$ represents the "standard" characterization of a vague concept and for a fuzzy set $f \in \mathscr{F}(X)$, we obtain that $R_{T}(f, g)=1$ or $R_{T}(g, f)=1$, we may say that $f$ is a $T$-outlier with respect to $g$. On the other hand, when $|\operatorname{supp} g|=|\operatorname{supp} f|=m$ and for all $p \in\{1, \ldots, m\}: R_{T}\left(f_{p}, g\right)=R_{T}\left(g_{p}, f\right)=0$, we may say that $f$ is $T$-consistent with $g$. Note that the statement " $g$ is $T$-superior to $f$ " can be also interpreted as " $f$ is $T$-inferior to $g$ ".

EXAMPLE 3. Assume that the standard (expected) progress on the set of four homework assignments in Example 1 is characterized by the fuzzy set $g$ given in Table 2, Consider also the progress of three students evaluated by the fuzzy sets $u, v$ and $w$ with the membership grades in Table 2.

TABle 2. Progress on $X$.

\begin{tabular}{|c||l|l|l|l|}
\hline Set & $x_{1}$ & $x_{2}$ & $x_{3}$ & \multicolumn{1}{|c|}{$x_{4}$} \\
\hline \hline$g$ & 0.6 & 0.55 & 0.7 & 0.8 \\
\hline$u$ & 0.95 & 0.85 & 1 & 1 \\
\hline$v$ & 0.4 & 0.2 & 0.3 & 0.45 \\
\hline$w$ & 0.55 & 0.55 & 0.7 & 0.65 \\
\hline
\end{tabular}

Then $R_{T_{M}}(g, u)=1$ and $R_{T_{M}}(v, g)=1$. Therefore, evaluations $u$ and $v$ are $T_{M}$-outliers with respect to $g$. We conclude that $u$ is $T_{M}$-superior and $v$ is $T_{M}$-inferior in comparison with the standard $g$. Because $|\operatorname{supp} g|=|\operatorname{supp} w|=4$ and for all $p \in\{1,2,3,4\}: R_{T_{M}}\left(w_{p}, g\right)=R_{T_{M}}\left(g_{p}, w\right)=0$, evaluation $w$ is $T_{M}$-consistent with $g$. After comparison of fuzzy sets $f_{A}$ and $f_{B}$ from Example 1 with the standard fuzzy set $g$ we realize that Benjamin's progress $\left(f_{B}\right)$ is $T_{M}$-inferior to the standard. Anton's progress $\left(f_{A}\right)$ is neither $T_{M}$-outlier nor $T_{M}$-consistent with the standard. If we use the coefficient $\delta_{T}$ defined by (3) as a measure of $T_{M}$-superiority, we obtain that $\delta_{T}\left(f_{A}, g\right)=1 / 3$. Therefore, the fuzzy set $g$ is $T_{M^{-}}$ superior to one third of the membership grades associated with the elements from the support of the fuzzy set $f_{A}$. 


\section{T-SUPERIORITY AND t-NORM-BASED IMAGES OF FUZZY SETS}

\section{T-images of fuzzy sets}

Assume $f, g \in \mathscr{F}(X)$. Any transformation of $f$ derived from a relationship between $f$ and $g$ will be called an image of $f$ with respect to $g$. A coarser image of $f$ reduces the total number of distinct membership grades of $f$. For a comparison of several fuzzy sets from $\mathscr{F}(X)$ with a given "standard" fuzzy set $g \in \mathscr{F}(X)$ we suggest comparison of coarser images of the considered fuzzy sets created with respect to $g$. For each $f \in \mathscr{F}(X)$ and for each $x \in X$, the image of $f(x)$ should reflect an evaluation of a relationship between $f(x)$ and the fuzzy set $g$. In this section we propose the $T$-image of $f$ induced by $g$, which, in a special case, can be considered as a soft evaluation of $T$-superiority of $g$ to $f$. In particular, for each $f(x), x \in \operatorname{supp} f$, we will look for the largest subset of large membership grades from $g$ whose $T$-aggregation will exceed $f(x)$. Further in the text, $g^{p}$ will denote the following restriction of $g$ to a $(k-p+1)$-element subset of $\operatorname{supp} g$ with the largest membership grades

$$
g^{p}=\left\{\left(x_{(p)}, g\left(x_{(p)}\right)\right), \ldots,\left(x_{(k)}, g\left(x_{(k)}\right)\right)\right\},
$$

where $x_{(p)}, x_{(p+1)} \ldots, x_{(k)}$ are reordered elements from supp $g$ such that $g\left(x_{(p)}\right) \leq$ $g\left(x_{(p+1)}\right) \leq \cdots \leq g\left(x_{(k)}\right)$.

Definition 4. Consider a t-norm $T$ and fuzzy sets $f, g \in \mathscr{F}(X), f \neq g, g \neq \mathbf{0}$, $f \neq \mathbf{1}$ and $|\operatorname{supp} g|=k$. Then the $T$ image of $f$ with respect to $g$ is the fuzzy set $\widetilde{f}(g, T)$ defined for all $x \in X$ as follows:

$$
\tilde{f}(g, T)(x)=0 \quad \text { if } \quad f(x)=0, \quad \text { or for all } \quad p \in\{1, \ldots, k\}
$$

we have that

$$
f(x) \geq T\left(\left\{g(z) ; z \in \operatorname{supp} g^{p}\right\}\right)
$$

otherwise

$$
\widetilde{f}(g, T)(x)=\frac{k-\left(i^{*}-1\right)}{k},
$$

where $i^{*}$ is the minimum of those $p \in\{1, \ldots, k\}$ for which $0<f(x)<T(\{g(z)$; $\left.\left.z \in \operatorname{supp} g^{p}\right\}\right)$.

Proposition 4. Let $f, g \in \mathscr{F}(X)$ and $|\operatorname{supp} g|=k$. Then for all $x \in X$ the membership grade $\tilde{f}(g, T)(x)$ satisfies the following properties:

i) when $f(x)>0$, then $\widetilde{f}(g, T)(x)=0$ if there is no restriction $g^{p}, p \in\{1, \ldots, k\}$ of the fuzzy set $g$ such that $f(x)<T\left(\left\{g(z) ; z \in \operatorname{supp} g^{p}\right\}\right)$,

ii) $\tilde{f}(g, T)(x)=1$ if and only if $f(x)<T(\{g(z) ; z \in \operatorname{supp} g\})$,

iii) if $h \in \mathscr{F}(X)$ and $h \leq f$, then $\widetilde{h}(g, T)(x) \geq \widetilde{f}(g, T)(x)$,

iv) if $h \in \mathscr{F}(X),|\operatorname{supp} h|=|\operatorname{supp} g|$ and $g \leq h$, then $\widetilde{f}(h, T)(x) \geq \widetilde{f}(g, T)(x)$. 
Proof.

i) For $f(x)>0$ we have that $\widetilde{f}(g, T)(x)=0$ if for all $p \in\{1, \ldots, k\}: f(x) \geq$ $T\left(\left\{g(z) ; z \in \operatorname{supp} g^{p}\right\}\right)$. This means that there is no restriction $g^{p}$ of $g$ such that $f(x)<T\left(\left\{g(z) ; z \in \operatorname{supp} g^{p}\right\}\right)$.

ii) $\tilde{f}(g, T)(x)=1$ if and only if $i^{*}=\min \{p \in\{1, \ldots, k\} ; f(x)<T(\{g(z)$; $\left.\left.\left.z \in \operatorname{supp} g^{p}\right\}\right)\right\}=1$ which means that $f(x)<T(\{g(z) ; z \in \operatorname{supp} g\})$.

iii) For all $x \in \operatorname{supp} f$ we have that $h(x) \leq f(x)$. If $h(x)>0$, then $s^{*}=\min \{p \in$ $\left.\{1, \ldots, k\} ; h(x)<T\left(\left\{g(x) ; x \in \operatorname{supp} g^{p}\right\}\right)\right\} \leq \min \{p \in\{1, \ldots, k\} ; f(x)<$ $\left.T\left(\left\{g(x) ; x \in \operatorname{supp} g^{p}\right\}\right)\right\}=i^{*}$. Therefore

$$
\widetilde{h}(g, T)(x)=\frac{k-\left(s^{*}-1\right)}{k} \geq \frac{k-\left(i^{*}-1\right)}{k}=\widetilde{f}(g, T)(x) .
$$

iv) When $g \leq h$ and $|\operatorname{supp} g|=|\operatorname{supp} h|=k$, then for all $p \in\{1, \ldots, k\}$ :

$$
\begin{aligned}
T\left(\left\{g(x) ; x \in \operatorname{supp} g^{p}\right\}\right)= & T\left(g\left(x_{(p)}\right), \ldots, g\left(x_{(k)}\right)\right) \leq \\
T\left(h\left(x_{(p)}\right), \ldots, h\left(x_{(k)}\right)\right) & =T\left(\left\{h(x) ; x \in \operatorname{supp} h^{p}\right\}\right) .
\end{aligned}
$$

Then

$$
\begin{aligned}
s^{*} & =\min \left\{p \in\{1, \ldots, k\} ; f(x)<T\left(\left\{g(x) ; x \in \operatorname{supp} g^{p}\right\}\right)\right\} \\
& \geq \min \left\{p \in\{1, \ldots, k\} ; f(x)<T\left(\left\{h(x) ; x \in \operatorname{supp} h^{p}\right\}\right)\right\}=i^{*} .
\end{aligned}
$$

Therefore

$$
\widetilde{f}(h, T)(x)=\frac{k-\left(i^{*}-1\right)}{k} \geq \frac{k-\left(s^{*}-1\right)}{k}=\widetilde{f}(g, T)(x) .
$$

Remark 3. The membership grade $\widetilde{f}(g, T)(x)$ is the relative cardinality of the support of the largest restriction $g^{p}$ of $g$ which is $T$-superior to the singleton $f(x)$. If $|\operatorname{supp} f| \leq|\operatorname{supp} g|$, the fuzzy set $\widetilde{f}(g, T)$ can be considered as a soft evaluation of $T$-superiority of $g$ to $f$.

Corollary 1. If $R_{T}(f, g)=1$, then for all $x \in \operatorname{supp} f$ we have $\tilde{f}(g, T)(x)=1$.

Corollary 2. Consider the lattice $(\mathscr{F}(X), \wedge, \vee, \mathbf{0}, \mathbf{1})$ and $f, h, g \in \mathscr{F}(X)$. Then for all $x \in X$ the following hold:

i) $\widetilde{(f \vee h})(g, T)(x)=\widetilde{f}(g, T)(x) \wedge \widetilde{h}(g, T)(x)$,

ii) $\widetilde{(f \wedge h})(g, T)(x)=\widetilde{f}(g, T)(x) \vee \widetilde{h}(g, T)(x)$.

Let us recall that for $\alpha \in(0,1]$ and $f \in \mathscr{F}(X)$, the $\alpha$-cut of $f$ is the crisp set $f_{\alpha}$ defined for all $x \in X$ by

$$
f_{\alpha}(x)= \begin{cases}1 & \text { if } f(x) \geq \alpha \\ 0 & \text { otherwise }\end{cases}
$$




\section{T-SUPERIORITY AND t-NORM-BASED IMAGES OF FUZZY SETS}

The concept of $\alpha$-cuts was generalized to $P$-level sets in [2] as follows.

Definition 5. Let $f \in \mathscr{F}(X)$ and $\Omega=\left\{t_{1}, \ldots, t_{k}\right\}$ be an arbitrary set of parameters from $(0,1)$. Then the $p$-level set of $f$ based on $\Omega$ is the fuzzy set $\Omega(f) \in \mathscr{F}(X)$ defined for all $x \in X$ by

$$
\Omega(f)(x)=\frac{|\{t \in \Omega ; t<f(x)\}|}{|\Omega|} .
$$

There is a connection between $T_{M}$ - images of fuzzy sets and $\alpha$-cuts ( $P$-level sets) of fuzzy sets.

Proposition 5. Let $f \in \mathscr{F}(X)$ such that $|\operatorname{supp} f|=X$ and $g$ be a constant function on $X$ with the level set $\Lambda_{g}=\{\alpha\}$. Then for all $x \in X$

$$
\tilde{f}\left(g, T_{M}\right)(x)=1-f_{\alpha}(x) .
$$

Pr o of. Because $|\operatorname{supp} f|=X,|\operatorname{supp} g|=1$ and $\Lambda_{g}=\{\alpha\}$ we have that

$$
\widetilde{f}\left(g, T_{M}\right)(x)= \begin{cases}0 & \text { if } f(x) \geq \alpha \\ 1 & \text { otherwise }\end{cases}
$$

Therefore $\widetilde{f}\left(g, T_{M}\right)(x)=1-f_{\alpha}(x)$.

In the case described in Proposition [5] the $T_{M}$-image of $f$ with respect to $g$ is the complement of the $\alpha$-cut of $f$, where $\{\alpha\}=\Lambda_{g}$.

Proposition 6. Let $f \in \mathscr{F}(X)$ and $|\operatorname{supp} f|=X$. Consider $g \in \mathscr{F}(X)$ such that $\Lambda_{f} \cap \Lambda_{g}=\emptyset, g(x)<1$ for all $x \in X$ and $\left|\Lambda_{g}\right|=k$. Set $\Omega_{g}=\Lambda_{g}=$ $\left\{t_{1}, t_{2}, \ldots, t_{k}\right\}, t_{1}<t_{2}<\cdots<t_{k}<1$. Then for all $x \in X$

$$
\widetilde{f}\left(g, T_{M}\right)(x)=1-\Omega_{g}(f)(x) .
$$

Pr o of. Because $|\operatorname{supp} f|=X$ and $\Lambda_{f} \cap \Lambda_{g}=\emptyset$, we obtain that $\tilde{f}\left(g, T_{M}\right)(x)=0$ if and only if $f(x)>t_{k}$. In this case

and therefore

$$
\Omega(f)(x)=\frac{\left|\left\{t \in \Omega_{g} ; t<f(x)\right\}\right|}{\left|\Omega_{g}\right|}=\frac{\left|\Omega_{g}\right|}{\left|\Omega_{g}\right|}=1,
$$

$$
\widetilde{f}\left(g, T_{M}\right)(x)=1-\Omega_{g}(f)(x) .
$$

Otherwise $\tilde{f}\left(g, T_{M}\right)(x)=\frac{k-\left(i^{*}-1\right)}{k}$, where $i^{*}$ is the minimum of those $p \in\{1, \ldots, k\}$ for which $0<f(x)<t_{p}$. Because $\Lambda_{f} \cap \Lambda_{g}=\emptyset$ we have that $k-\left(i^{*}-1\right)=\mid\left\{t \in \Omega_{g}\right.$; $t>f(x)\} \mid$. Then

$$
\widetilde{f}\left(g, T_{M}\right)(x)=1-\frac{\left|\left\{t \in \Omega_{g} ; t<f(x)\right\}\right|}{\left|\Omega_{g}\right|}=1-\Omega_{g}(f)(x) .
$$

Because of (10) and (11) we conclude that $\widetilde{f}\left(g, T_{M}\right)(x)=1-\Omega_{g}(f)(x)$. 
In the case described in Proposition [6] the $T_{M}$-image of $f$ with respect to $g$ is the complement of the $P$-level set of $f$ based on $\Omega_{g}$.

Remark 4. Let $|\operatorname{supp} g|=k$. Then for arbitrary t-norm $T$ and for any fuzzy set $f \neq g$ we have that $\widetilde{f}(g, T): X \rightarrow\{0,1 / k, 2 / k, \ldots,(k-1) / k, 1\}$.

ExAmple 4 . Let $X=\left\{x_{1}, \ldots, x_{6}\right\}$. Assume fuzzy sets $f_{C}, f_{D}, g \in \mathscr{F}(X)$ with membership functions given in Table 3. $T_{m}$-images and $T_{p}$-images of $f_{C}$ and $f_{D}$ with respect to $g$ are presented in Table 4. Though the membership grades of $f_{C}$ and $f_{D}$ are different, their $T_{M}$ images are indistinguishable. Therefore, in a quick comparison, when aggregation by $T_{M}$ is used, $f_{C}$ and $f_{D}$ may be considered equal with respect to the "standard" described by $g$. For example, if $g$ represents the standard progress on $X$, as described in Example 3. performance characterized by $f_{C}$ and $f_{D}$ may be reported as "the same" when compared to the standard. However, when aggregation by $T_{p}$ is used, $T_{p}$ images of $f_{C}$ and $f_{D}$ revealed some differences in membership grades of $x_{1}$ and $x_{6}$.

TABle 3. Membership function of selected fuzzy sets.

\begin{tabular}{|c||l|c|c|c|c|l|}
\hline Set & $x_{1}$ & $x_{2}$ & $x_{3}$ & $x_{4}$ & $x_{5}$ & $x_{6}$ \\
\hline \hline$f_{C}$ & 0.2 & 0.5 & 0.58 & 0.7 & 0.28 & 0.6 \\
\hline$f_{D}$ & 0.28 & 0.4 & 0.56 & 0.65 & 0.27 & 0.68 \\
\hline$g$ & 0.3 & 0.6 & 0.55 & 0.6 & 0.8 & 0.8 \\
\hline
\end{tabular}

TABLE 4. Membership grades of selected $T$-images.

\begin{tabular}{|c||c|c|c|c|c|c|}
\hline Set & $x_{1}$ & $x_{2}$ & $x_{3}$ & $x_{4}$ & $x_{5}$ & $x_{6}$ \\
\hline$\widetilde{f_{C}}\left(g, T_{M}\right)$ & 1 & $5 / 6$ & $4 / 6$ & $2 / 6$ & 1 & $2 / 6$ \\
\hline$\widetilde{f_{D}}\left(g, T_{M}\right)$ & 1 & $5 / 6$ & $4 / 6$ & $2 / 6$ & 1 & $2 / 6$ \\
\hline$\widetilde{f_{C}}\left(g, T_{P}\right)$ & $4 / 6$ & $2 / 6$ & $2 / 6$ & $1 / 6$ & $3 / 6$ & $2 / 6$ \\
\hline$\widetilde{f_{D}}\left(g, T_{P}\right)$ & $3 / 6$ & $2 / 6$ & $2 / 6$ & $1 / 6$ & $3 / 6$ & $1 / 6$ \\
\hline
\end{tabular}

\section{Conclusion}

Our work is a contribution to applications of t-norms in analysis of vague data described by fuzzy sets. We suggested an evaluation of a rare relationship between two fuzzy sets, when one of them can be considered "superior" with respect to the other. We introduced the notion of T-superiority which may help 


\section{T-SUPERIORITY AND t-NORM-BASED IMAGES OF FUZZY SETS}

in decision making to identify outliers among fuzzy sets under consideration. Then we proposed a comparison of several evaluations of the same vague concept with a given standard evaluation of the concept based on the $T$-images of fuzzy sets. In our future work we will study the relationship between $T$-images and approximations of fuzzy sets by rough sets [8].

\section{REFERENCES}

[1] BELIAKOV, G.-PRADERA, A.-CALVO, T.: Aggregation Functions: A Guide for Practitioners. Springer-Verlag, New York, 2007.

[2] BODJANOVA, S.-KALINA, M.: Coarsening of fuzzy sets, in: Proc. of the 11th Internat. Symp. on Intelligent Syst. and Inform.-SISY '13, Subotica, Serbia, 2013, IEEE, 2013, pp. 51-56.

[3] Aggregation operators (Calvo, T., Mayor, G. and Mesiar, R., eds.), Physica-Verlag, Heidelberg, 2002.

[4] FUNDAMENTALS OF FUZZY SETS (Dubois, D. and Prade, H., eds.) Kluwer, Dordrecht, 2000.

[5] GRABISCH, M.-PAP, E.-MARICHAL, J. L.-MESIAR, R.: Aggregation Functions. University Press, Cambridge, 2009.

[6] KLEMENT, E.P.-MESIAR, R.-PAP, E.: Triangular norms. Kluwer, Dordrecht, 2000.

[7] KLIR, G. J.-FOLGER, T.: Fuzzy Sets, Uncertainty and Information. Prentice Hall, Englewood Cliffs, 1988.

[8] PAWLAK, Z.: Rough Sets, Theoretical Aspects of Reasoning about Data. Kluwer, Dordrecht, 1991.

[9] RADECKI, T.: Level-fuzzy sets, J. Cybernet. 7 (1977), 189-198.

[10] ZADEH, L.: Fuzzy sets, Inform. and Control 8 (1965), 338-353.

Received July 15, 2016
Slavka Bodjanova

Texas A\&M University-Kingsville

Department of Mathematics, MSC 172

Kingsville TX 78363

U.S.A.

E-mail: kfsb000@tamuk.edu

Slovak University of Technology in Bratislava

Faculty of Civil Engineering

Dept. of Mathematics

Radlinského 11

SK-810-05 Bratislava

SLOVAKIA

E-mail: kalina@math.sk 